Original article

\title{
Contribution of catastrophizing to disability and pain intensity after osteoporotic vertebral compression fracture
}

\author{
Ho-Joong Kim ${ }^{a}$, Yun-Ho Kim ${ }^{a}$, Kyoung-Tak Kang ${ }^{b}$, Bong-Soon Chang ${ }^{c}$, Choon-Ki Lee ${ }^{c}$, \\ Jin S. Yeom ${ }^{\text {a, * }}$ \\ a Spine Center and Department of Orthopaedic Surgery, Seoul National University College of Medicine, Seoul National University Bundang Hospital, 166 \\ Gumiro, Bundang-gu, Sungnam 463-707, Republic of Korea \\ ${ }^{\mathrm{b}}$ Department of Mechanical Engineering, Yonsei University, 134 Shinchon-dong, Seodaemun-gu, Seoul, Republic of Korea \\ c Department of Orthopaedic Surgery, Seoul National University College of Medicine, Seoul National University Hospital, 101 Daehangno, Jongno-gu, Seoul \\ 110-744, Republic of Korea
}

\section{A R T I C L E I N F O}

\section{Article history:}

Received 13 October 2015

Received in revised form 12 January 2016

Accepted 16 February 2016

Available online $\mathrm{xxx}$

\begin{abstract}
A B S T R A C T
Background: Pain catastrophizing is a key variable that contributes to disability not only in chronic pain disorders but also after trauma. However, there is little evidence concerning the effect of catastrophizing on pain intensity and disability after osteoporotic vertebral compression fracture. Therefore, the purpose of this study was to evaluate the contribution of catastrophizing to disability and pain intensity after osteoporotic vertebral compression fracture.

Method: We analyzed 35 patients with acute single-level osteoporotic vertebral compression fractures within 3 days of trauma. Data on demographics, education level, Charlson comorbidity index, pain catastrophizing scale (PCS) score, visual analog scale (VAS) score for back pain, and Oswestry Disability Index (ODI) were collected. VAS score for back pain and ODI were assessed at enrollment as well as at 2 , 6 , and 12 weeks after fracture.

Results: Each VAS score for back pain and ODI significantly improved compared to the initial values $(\mathrm{P}<0.001)$. Among the independent variables, age and/or PCS score significantly correlated with VAS score for back pain and/or ODI over follow-up assessments. Hierarchical regression analysis finally showed that PCS score was a significant predictor for disability only in the acute period such as immediately and 2 weeks after fracture, whereas age was significantly associated with ODI at 6 and 12 weeks after fracture.

Conclusions: The present study shows that catastrophizing can contribute to disability only in the acute period after osteoporotic vertebral compression fracture. As the compression fracture heals, however, age is the critical determinant of disability.
\end{abstract}

(ङ) 2016 The Japanese Orthopaedic Association. Published by Elsevier B.V. All rights reserved.

\section{Introduction}

Pain catastrophizing is an adverse coping mechanism that involves an exaggerated negative orientation to actual or anticipating pain and is related to greater pain intensity, pain chronicity, and anxiety $[1,2]$. Recent burgeoning research has emphasized its role in chronic pain-related treatment outcomes [3-6], and a higher level of catastrophizing is considered a poor prognostic factor for both conservative treatment and surgical intervention [3,5,7-11].

\footnotetext{
* Corresponding author. Tel.: +82 317877195 .

E-mail address: highcervical@gmail.com (J.S. Yeom).
}

As the age of the population increases, osteoporotic compression fracture represents a growing serious socioeconomic burden on public and personal health. Although of its benign characteristic, osteoporotic compression fracture may cause intractable pain, disability, and diminished quality of life [12-14]. In addition, a recent prospective study showed that the mental aspect of a patient's general health status deteriorates despite fracture healing [15]. Considering the significance of catastrophizing in pain and disability, it can be assumed that pain and disability caused by osteoporotic compression fracture would be influenced by catastrophizing. Moreover, if catastrophizing would increase pain and disabilities after osteoporotic compression fracture, the proper management of catastrophizing should be important. To our 
knowledge, however, no study has examined the impact of catastrophizing on pain and disability after osteoporotic compression fracture. We hypothesized that catastrophizing could influence the improvement of pain and disability after osteoporotic compression fracture. Hence, we aimed to investigate the influence of pain catastrophizing-measured using the pain catastrophizing scale (PCS)-on pain intensity and disability after osteoporotic compression fracture [16].

\section{Materials and methods}

\subsection{Study design and patients}

This observational study was approved by the hospital institutional review board. All data, including the PCS data, were prospectively collected as part of the routine care of patients with compression fracture and, analyzed retrospectively. The inclusion criteria were as follows: 1 ) age $\geq 50$ years; and 2) the presence of back pain due to acute compression fracture without neurological deficit within 3 days of minor trauma at a single level. Compression fracture was defined as an axial compression of only the anterior column of the vertebral body with intact posterior elements confirmed on magnetic resonance imaging. The exclusion criteria included a history of more than two recent vertebral fractures, malignant compression fracture, neurological compromise, nonambulatory state before the fracture, previous injury or surgery at the fractured level, inability to complete the questionnaires on pain and disability, and history of major psychiatric disorders such as schizophrenia and major depression. The present observational study was performed at the spinal center of a tertiary care teaching institution between June 2014 and December 2014.

\subsection{Treatment}

Patients were strictly maintained on bed rest until a TLSO was applied (How-medicare, Seoul, Korea). Braces were required to be worn at all times except when lying down. All patients were instructed to wear the brace for a total of 8 weeks. All patients took pain medication as necessary, and were counseled on restricting the bending of the spine, heavy lifting, and carrying during the first 8 weeks. After 8 weeks, a 2-week weaning period was initiated.

\subsection{Measurement of catastrophizing}

The PCS was used to measure pain-related catastrophizing [16,17]. The PCS comprises 13 items describing different thoughts and feelings that individuals may experience while in pain (Table 1)

\section{Table 1}

Pain catastrophizing scale.

\begin{tabular}{ll}
\hline Item & \\
\hline Helplessness & 1. I worry all the time about whether the pain will end \\
2. I feel I can't go on & 3. It's terrible and I think it's never going to get any better \\
4. It's awful and I feel that it overwhelms me \\
5. I feel I can't stand it any more \\
12. There is nothing I can do to reduce the intensity of the \\
pain \\
6. I become afraid that the pain may get worse \\
7. I think of other painful experiences \\
13. I wonder whether something serious may happen \\
8. I anxiously want the pain to go away \\
9. I can't seem to keep it out of my mind \\
10. I keep thinking about how much it hurts \\
11. I keep thinking about how badly I want the pain to stop
\end{tabular}

[16]. Subjects were asked to reflect on painful experiences and indicate the degree to which they experienced each of the 13 thoughts or feelings when in pain on a 5-point scale ranging from 0 ("not at all") to 4 ("always"). The PCS yields a total score and 3 subscale scores that assess rumination, magnification, and helplessness. Higher PCS scores indicate greater pain catastrophizing.

\subsection{Data measurement and clinical outcome assessment}

A blinded clinical research assistant collected data on the demographics, education level, Charlson comorbidity index, PCS score, visual analog scale (VAS) score for back pain, and Oswestry Disability Index (ODI). The level of education was estimated by the highest qualification achieved. Thus, patients were allocated to one of the following 4 groups: elementary school graduation (elementary group), junior high school graduation (junior group), senior high school graduation (senior group), or college/university graduation (college group) [18]. Comorbidity was assessed using a validated comorbidity measure, the Charlson comorbidity index [19]. The Charlson comorbidity index consists of a weighted scale of 17 comorbidities including cardiac, pulmonary, renal, and hepatic disease; diabetes; cancer; and HIV, expressed as a summative score [19]. At 2, 6, and 12 weeks after the compression fracture, the VAS score for back pain and ODI were re-assessed. The ODI is a self-administered questionnaire measuring back-specific function. The questionnaire comprises 10 items, each with 6 response levels. Each item is scored from 0 to 5 , and the total score is converted to a $0-100$ scale [20]. The VAS for back pain comprises a 10-cm line with "none" (0) on one end and "disabling pain" [10] on the other end. Patients were asked to place a mark on the $10-\mathrm{cm}$ line to represent their perceived level of back pain. The measured distance $(\mathrm{cm})$ from their mark to zero was considered the VAS score.

\subsection{Statistical analysis}

The calculated sample size showed that a minimum of 35 patients was necessary to achieve a power of $80 \%$ to detect an $\mathrm{r}^{2}=0.35$ on linear multiple regression with 6 predictors $(\alpha=0.05$, $\beta=0.2$ ). Over each follow-up assessment, the mean VAS score for back pain and ODI were compared to the initial VAS score for back pain and ODI using a paired $t$-test. The correlations between ODI and VAS score for back pain and demographics including age, sex, education level, and body mass index (BMI) over follow-up assessments (from immediately to 12 weeks after fracture) were analyzed by Pearson correlations for continuous variables and oneway analysis of variance for categorical variables. The variables that were significantly associated with VAS for back pain and ODI on the correlation analysis were entered into the multiple linear regression models with potentially important variables such as age, BMI, and sex regardless of statistical significance. For the multiple linear regression models, we anticipated a potential issue of collinearity between the variables and set an a priori rule to exclude variables with correlation coefficients $\geq 0.50$. At each time point during the follow-up assessment (immediately and 2, 6, and 12 weeks after fracture), hierarchical multiple regression analyses were performed to control for the effect of covariates which could reveal the effects of the potential predictors independent of the influence of the covariates. Based on the correlation between variables at each assessment time, confounding variables were entered in the first step. Secondly, the main predictor (PCS score or age) was entered in the regression model. All statistical analyses were performed using the SPSS 20.0.0 statistics package (SPSS, Inc., Chicago, IL). The alpha level of significance was set at 0.05 . 


\section{Results}

\subsection{Patient characteristics}

A total of 42 patients were initially assessed for eligibility between June 2014 and December 2014. After 7 patients were excluded, a total of 35 patients were included in this study (Fig. 1). Mean age and $\mathrm{BMI} \pm$ standard deviation $(\mathrm{SD})$ were $71.86 \pm 9.33$ and $23.05 \pm 2.85$, respectively. Initial mean VAS score for back pain and $\mathrm{ODI} \pm$ SD were $8.06 \pm 2.00$ and $70.00 \pm 13.99$, respectively (Table 2 ). Mean PCS score \pm SD was $25.52 \pm 11.40$. For pain medication, 15 and 18 patients used weak opioids at 2 and 6 weeks after fracture, respectively.

\subsection{Changes in VAS score for back pain and ODI}

Over the follow-up assessment, VAS score for back pain and ODI were significantly decreased compared to each initial value. The VAS score for back pain \pm SD significantly decreased to $6.00 \pm 1.8$ $(\mathrm{P}<0.001), 4.08 \pm 2.08(\mathrm{P}<0.001)$, and $3.07 \pm 2.12(\mathrm{P}<0.001)$ at 2 , 6 , and 12 weeks after fracture, respectively, compared to the initial values. The ODI \pm SD also significantly decreased to $53.46 \pm 14.01$ $(\mathrm{P}<0.001), 40.70 \pm 13.16(\mathrm{P}<0.001)$, and $30.56 \pm 16.09(\mathrm{P}<0.001)$ at 2,6 , and 12 weeks after fracture, respectively, compared to the initial values (Fig. 2).

\subsection{Correlations among variables in both groups}

There was multicollinearity among variables including age, VAS score for back pain, ODI, and PCS score (Table 3). In the acute stage (immediately and 2 weeks after the compression fracture), PCS significantly correlated with VAS score for back pain and ODI, whereas in the 12 weeks after compression fracture, age significantly correlated with VAS score for back pain and ODI (Table 3). Furthermore, at each assessment time, there was a significant correlation between VAS score for back pain and ODI $(r=0.447$, $\mathrm{P}=0.009$ immediately after fracture; $\mathrm{r}=0.724, \mathrm{P}<0.0012$ weeks after fracture; $\mathrm{r}=0.514, \mathrm{P}=0.0076$ weeks after fracture; and $\mathrm{r}=0.664, \mathrm{P}<0.00112$ weeks after fracture).

\subsection{Hierarchical multiple regression for ODI and VAS score for back pain}

Tables 4 and 5 show all the regression models for explaining back pain and disability at each assessment time after osteoporotic vertebral compression fracture, respectively. Given the multicollinearity and potential significance of the variables, PCS score, age, sex, BMI, education level, and Charlson comorbidity index were entered in the model.

Immediately and 2 weeks after fracture, age, sex, BMI, education level, and Charlson comorbidity index in step 1 accounted for $28.4 \%$

42 patients were assessed for eligibility

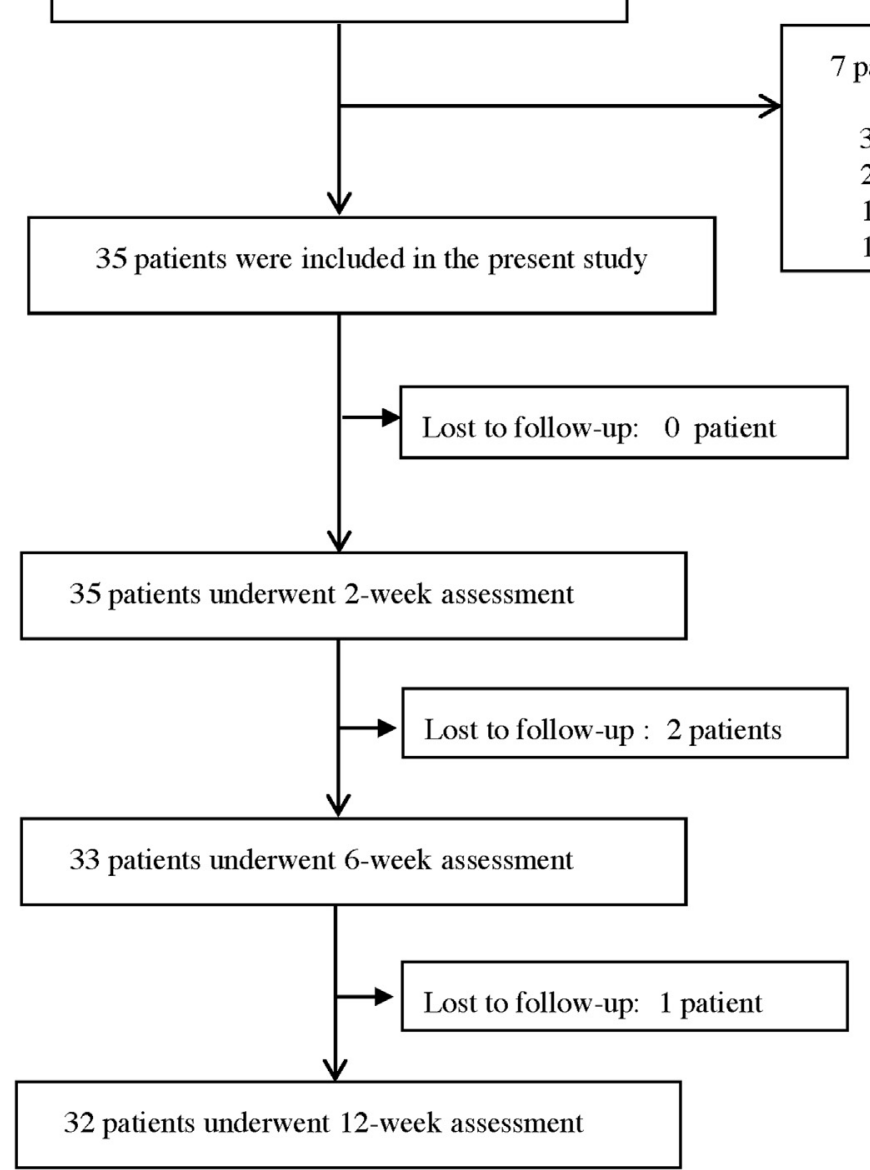

patients were excluded

3 patient was not literate

2 patients had multiple-level fractures

1 patients had surgeries at the fractured level

1 patients had major depression

Fig. 1. Enrollment and follow-up of the observational cohort. 
Table 2

Descriptive statistics of the subjects in the study. Values are mean \pm SD.

\begin{tabular}{ll}
\hline No. & 35 patients \\
\hline Age (years) & $71.86 \pm 9.33$ \\
Male:female (numbers) & $5: 30$ \\
BMI $\left(\mathrm{kg} / \mathrm{cm}^{2}\right)$ & $23.05 \pm 2.85$ \\
BMD $\left(\mathrm{g} / \mathrm{cm}^{2}\right)$ & \\
Total lumbar & $0.90 \pm 0.16$ \\
Femur neck & $0.70 \pm 0.11$ \\
Total hip & $0.75 \pm 0.11$ \\
VAS for back pain & $8.06 \pm 2.00$ \\
ODI & $70.00 \pm 13.99$ \\
The level of education (numbers) & \\
Elementary & 6 \\
Junior high school & 8 \\
Senior high school & 16 \\
College or university & 5 \\
Pain catastrophizing scale (total) & $25.52 \pm 11.40$ \\
Helplessness & $9.78 \pm 6.44$ \\
Magnification & $4.48 \pm 2.78$ \\
Rumination & $11.27 \pm 3.72$ \\
Level (n) & \\
T 7 & 1 \\
T8 & 1 \\
T 9 & 3 \\
T10 & 7 \\
T11 & 8 \\
T12 & 9 \\
L1 & 4 \\
L2 & 1 \\
L3 & 1 \\
\hline
\end{tabular}

BMI; body mass index, VAS; Visual Analog Pain Scale, ODI; Oswestry Disability Index, SD; standard deviation.

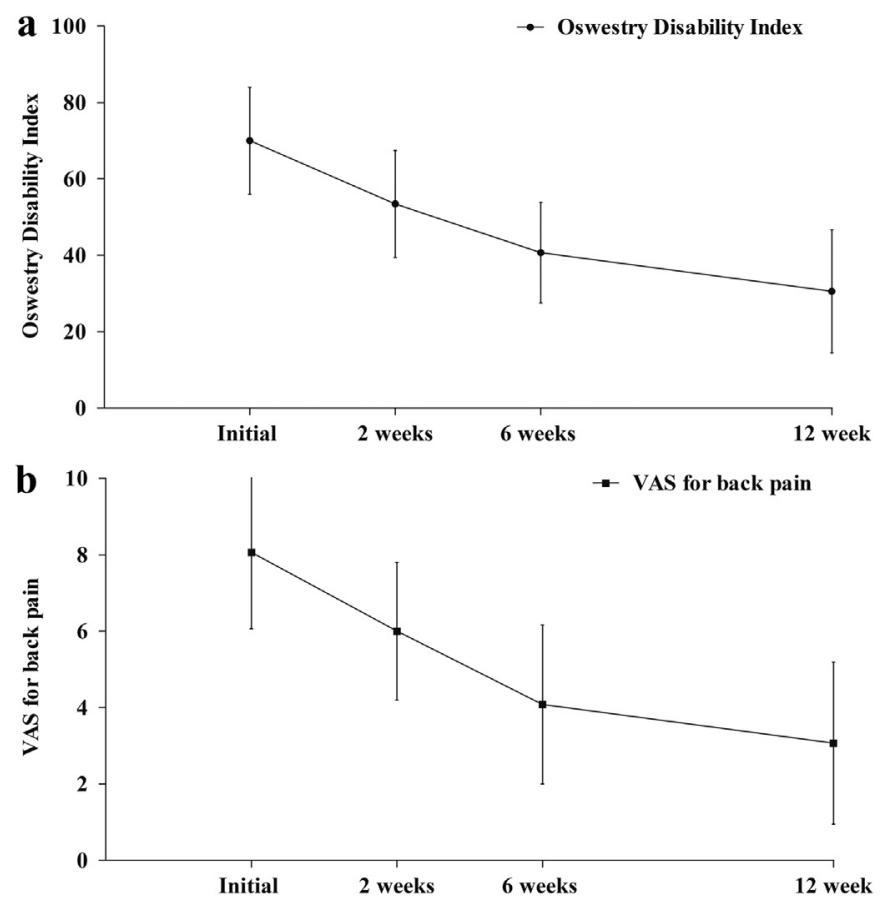

Fig. 2. Treatment outcomes at the follow-up assessment points (2, 6, and 12 weeks after trauma). Error bars indicate standard deviation. a. Oswestry Disability Index (ODI), b. Visual analog scale (VAS) score for back pain.

$(\mathrm{P}=0.090)$ and $13.6 \%(\mathrm{P}=0.635)$ of the predictions of VAS score for back pain, respectively. When PCS score was entered in step 2, these models accounted for $35.4 \%(P=0.054)$ and $33.5 \%(P=0.156)$ of the prediction of VAS score for back pain immediately and 2 weeks after fracture, respectively. However, the models' results were not statistically significant, and only PCS score had a significant association with VAS score for back pain at 2 weeks after fracture (standardized $\beta=0.477, \mathrm{P}=0.021$ ) (Table 4). For the prediction of ODI immediately and 2 weeks after fracture, age, sex, BMI, education level, and Charlson comorbidity index included in step 1 accounted for $20.2 \%(P=0.267)$ and $35.8 \%$ (0.066), respectively. When PCS score was entered in step 2, the accounted for 39.7\% $(\mathrm{P}=0.029)$ and $54.0 \%(\mathrm{P}=0.007)$ of the predicted variance of ODI immediately and 2 weeks after fracture, respectively, and the multiple regression model findings were statistically significant (Table 5). Hierarchical linear regression analysis with PCS score as an independent variable controlling for the effects of demographic variables, education level, and Charlson comorbidity index indicated that ODI was significantly predicted by PCS (standardized $\beta=0.490, P=0.008$, immediately after fracture; standardized $\beta=0.456, \mathrm{P}=0.009,2$ weeks after fracture) (Table 5).

At 6 weeks after fracture, age, sex, BMI, education level, and Charlson comorbidity index included in step 1 accounted for $11.9 \%$ $(\mathrm{P}=0.782)$ of the VAS score for back pain. PCS was entered in step 2 , which contributed to $27.3 \%(P=0.421)$ of the VAS score for back pain at 6 weeks after fracture. Considering correlations between VAS score for back pain at 12 weeks after fracture and other variables, PCS score, sex, BMI, education level, and Charlson comorbidity index were included in step 1, which accounted for $15.2 \%$ $(\mathrm{P}=0.567)$ of the VAS score for back pain at 12 weeks after fracture. Additional inclusion of age in step 2 contributed to $23.0 \%$ $(\mathrm{P}=0.425)$ of the predicted variance. However, the models were not statistically significant, and any variables did not explain the VAS score for back pain at 6 weeks and 12 weeks after fracture (Table 4). For prediction of ODI at 6 weeks and 12 weeks after fracture, PCS score, sex, BMI, education level, and Charlson comorbidity index included in step 1 accounted for $17.4 \%(P=0.590)$ and $24.2 \%(P=0.285)$, respectively. Age was entered at step 2 , which contributed to $36.1 \%(P=0.207)$ and $53.8 \%(P=0.010)$ to the predicted variance of ODI at 6 and 12 weeks after fracture, respectively. Therefore, the final step 2 model for the prediction of ODI 12 weeks after fracture was statistically significant. Hierarchical regression analysis including age showed that age was significantly associated with ODI after 6 weeks (standardized $\beta=0.538$; $\mathrm{P}=0.040$ ) and 12 weeks (standardized $\beta=0.719 ; \mathrm{P}=0.002$ ) (Table 5).

\section{Discussion}

The present study shows that catastrophizing can contribute to severity of disability only in the early stage after compression fracture. As the fracture healed, patient age was the critical determinant of low back pain and disability. This finding indicates that the contribution of catastrophic thinking to disability after vertebral compression fracture differs from that of various chronic pain disorders $[3,4,10,18,21-23]$.

We found that catastrophizing was a key determinant of disability within 2 weeks after vertebral compression fracture. This agrees well with results from previous studies [10,11,21,24], which have consistently shown that catastrophizing is a powerful marker of heightened pain experience and disability. After skeletal trauma, catastrophizing is the most notable individual predictor of disability and pain [10]. Even in patients with degenerative spinal disease, a high level of catastrophizing in response to pain may be associated with chronic low back pain and disability $[2,18]$. Therefore, the present results extend previous research by showing that a coping style represented by magnification, rumination, and feelings of helplessness known as catastrophizing may be a primary risk factor for short-term disability following osteoporotic vertebral compression fracture. In addition, this suggests the importance of 
Table 3

Correlations among variables and VAS for back pain/ODI score.

\begin{tabular}{|c|c|c|c|c|c|c|c|c|c|c|c|c|}
\hline \multirow[t]{2}{*}{ Variables } & \multicolumn{2}{|l|}{ Age } & \multicolumn{2}{|l|}{ Gender } & \multicolumn{2}{|l|}{ BMI } & \multicolumn{2}{|c|}{$\begin{array}{l}\text { Education } \\
\text { attainment }\end{array}$} & \multicolumn{2}{|l|}{ PCS } & \multicolumn{2}{|c|}{$\begin{array}{l}\text { Charlson } \\
\text { comorbidity index }\end{array}$} \\
\hline & $r$ & $\mathrm{p}$ & $r$ & $\mathrm{p}$ & $r$ & $\mathrm{p}$ & $r$ & $\mathrm{p}$ & $r$ & $\mathrm{p}$ & $\mathrm{r}$ & $\mathrm{p}$ \\
\hline \multicolumn{13}{|c|}{ VAS for back pain } \\
\hline Initial & 0.427 & 0.013 & -0.202 & 0.206 & -0.176 & 0.326 & -0.322 & 0.068 & 0.388 & 0.026 & 0.269 & 0.130 \\
\hline 2 weeks & 0.241 & 0.199 & 0.001 & 0.986 & 0.074 & 0.696 & -0.192 & 0.308 & 0.488 & 0.008 & 0.218 & 0.247 \\
\hline 6 weeks & 0.166 & 0.419 & 0.008 & 0.971 & 0.264 & 0.193 & -0.257 & 0.205 & 0.451 & 0.027 & -0.057 & 0.478 \\
\hline 12 weeks & 0.385 & 0.036 & 0.057 & 0.764 & 0.045 & 0.812 & -0.309 & 0.097 & 0.292 & 0.132 & 0.104 & 0.586 \\
\hline \multicolumn{13}{|l|}{ ODI } \\
\hline Initial & 0.184 & 0.305 & -0.233 & 0.192 & -0.321 & 0.068 & -0.105 & 0.560 & 0.495 & 0.003 & 0.053 & 0.769 \\
\hline 2 weeks & 0.544 & 0.002 & 0.148 & 0.434 & 0.051 & 0.790 & -0.352 & 0.057 & 0.489 & 0.008 & 0.210 & 0.266 \\
\hline 6 weeks & 0.581 & 0.002 & 0.190 & 0.352 & 0.261 & 0.198 & -0.136 & 0.506 & 0.285 & 0.177 & 0.078 & 0.706 \\
\hline 12 weeks & 0.698 & $<0.001$ & 0.155 & 0.422 & 0.103 & 0.595 & -0.305 & 0.108 & 0.372 & 0.056 & 0.073 & 0.706 \\
\hline
\end{tabular}

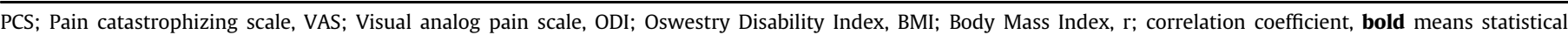
significance.

Table 4

Results of hierarchical multivariate regression models for prediction of VAS for back pain.

\begin{tabular}{|c|c|c|c|c|c|}
\hline & & $\mathrm{R}^{2}$ & $\mathrm{~F}$ & Standardized $\beta$ & $P$ \\
\hline \multirow[t]{5}{*}{ Immediately } & Step 1 & 0.284 & 2.146 & & 0.090 \\
\hline & Sex, age, BMI, educational attainment, Charlson comorbidity index & & & & \\
\hline & Step 2 & 0.354 & 2.379 & & 0.054 \\
\hline & Sex, age, BMI, educational attainment, Charlson comorbidity index & & & & \\
\hline & PCS & & & 0.294 & 0.105 \\
\hline \multirow[t]{5}{*}{2 weeks } & Step 1 & 0.136 & 0.692 & & 0.635 \\
\hline & Sex, age, BMI, educational attainment, Charlson comorbidity index & & & & \\
\hline & Step 2 & 0.335 & 1.761 & & 0.156 \\
\hline & Sex, age, BMI, educational attainment, Charlson comorbidity index & & & & \\
\hline & PCS & & & 0.477 & 0.021 \\
\hline \multirow[t]{5}{*}{6 weeks } & Step 1 & 0.119 & 0.487 & & 0.782 \\
\hline & Sex, age, BMI, educational attainment, Charlson comorbidity index & & & & \\
\hline & Step 2 & 0.273 & 1.064 & & 0.421 \\
\hline & Sex, age, BMI, educational attainment, Charlson comorbidity index & & & & \\
\hline & PCS & & & 0.429 & 0.075 \\
\hline \multirow[t]{5}{*}{12 weeks } & Step 1 & 0.152 & 0.791 & & 0.567 \\
\hline & PCS, age, BMI, educational attainment, Charlson comorbidity index & & & & \\
\hline & 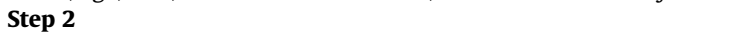 & 0.230 & 1.045 & & 0.425 \\
\hline & PCS, age, BMI, educational attainment, Charlson comorbidity index & & & & \\
\hline & Age & & & 0.377 & 0.161 \\
\hline
\end{tabular}

PCS; Pain catastrophizing scale, VAS; Visual analog pain scale, ODI; Oswestry Disability Index, BMI; Body Mass Index, bold means statistical significance.

Table 5

Results of hierarchical multivariate regression models for prediction of ODI after compression fracture.

\begin{tabular}{|c|c|c|c|c|c|}
\hline & & $\mathrm{R}^{2}$ & $\mathrm{~F}$ & Standardized $\beta$ & $P$ \\
\hline \multirow[t]{5}{*}{ Immediately } & Step 1 & 0.202 & 1.369 & & 0.267 \\
\hline & Sex, age, BMI, educational attainment, Charlson comorbidity index & & & & \\
\hline & Step 2 & 0.397 & 2.847 & & $\mathbf{0 . 0 2 9}$ \\
\hline & Sex, age, BMI, educational attainment, Charlson comorbidity index & & & & \\
\hline & PCS & & & 0.490 & 0.008 \\
\hline \multirow[t]{5}{*}{2 weeks } & Step 1 & 0.358 & 2.451 & & 0.066 \\
\hline & Sex, age, BMI, educational attainment, Charlson comorbidity index & & & & \\
\hline & Step 2 & 0.540 & 4.101 & & 0.007 \\
\hline & Sex, age, BMI, educational attainment, Charlson comorbidity index & & & & \\
\hline & PCS & & & 0.456 & 0.009 \\
\hline \multirow[t]{5}{*}{6 weeks } & Step 1 & 0.174 & 0.760 & & 0.590 \\
\hline & Sex, PCS, BMI, educational attainment, Charlson comorbidity index & & & & \\
\hline & Step 2 & 0.361 & 1.602 & & 0.207 \\
\hline & Sex, age, BMI, educational attainment, Charlson comorbidity index & & & & \\
\hline & Age & & & 0.538 & 0.040 \\
\hline \multirow[t]{5}{*}{12 weeks } & Step 1 & 0.242 & 1.344 & & 0.285 \\
\hline & Sex, PCS, BMI, educational attainment, Charlson comorbidity index & & & & \\
\hline & Step 2 & 0.230 & 3.24 & & 0.010 \\
\hline & PCS, age, BMI, educational attainment, Charlson comorbidity index & & & & \\
\hline & Age & & & 0.719 & 0.002 \\
\hline
\end{tabular}

PCS; Pain catastrophizing scale, VAS; Visual analog pain scale, ODI; Oswestry Disability Index, BMI; Body Mass Index, bold means statistical significance. 
management of pain catastrophizing such as cognitive behavior treatment in acute period of compression fracture.

This study revealed that 6 and 12 weeks after vertebral compression fracture, catastrophizing no longer influenced low back pain and disability. This finding seems to differ from that of a previous study that reported that catastrophizing could contribute to disability in the early and late periods after skeletal trauma [10]. We think that this difference is attributable to the difference in injury severity between studies. While the present study focused on only benign vertebral compression caused by minor trauma, one previous study focused on skeletal injuries of varying severity (1-6 on the Abbreviated Injury Scale) [25]. Therefore, it can be speculated that in the case of minor traumatic injury such as osteoporotic compression fracture, catastrophizing influences pain intensity and severity only in the early stage after fracture, that is, before fracture healing occurs. This finding also supports that of a previous study that demonstrated that there was no correlation between catastrophizing and disability 6 weeks after distal radial fracture [26]. In contrast, catastrophizing significantly correlated with upper extremity-specific disability in various diseases including trigger finger, carpal tunnel syndrome, Dupuytren contracture, de Quervain syndrome, wrist ganglion cyst, and lateral epicondylosis [26]. Therefore, it can be assumed that catastrophizing could still influence disability several weeks after trauma in case of severe vertebral fracture with neurological compromise because chronic pain remains in this case.

Another important issue about catastrophizing in response to pain is its causal relationship with pain intensity and disability. Although the present study's design was longitudinal and not cross-sectional, we did not re-assess PCS score over the follow-up period. Therefore, the correlational nature of the data in this study does not allow conclusions to be drawn concerning the influence of pain on catastrophizing and vice-versa. This is the limitation of the present study. In fact, the issues of whether the pain experience can be influenced by changes in catastrophizing and whether changes in the pain experience result in changes in catastrophizing may persist. However, catastrophizing is considered to have a causal or antecedent correlation with pain [1]. Furthermore, although catastrophizing was associated with disability in the acute period after compression fracture, this was not a significant predictor of VAS score for back pain even in the acute period of compression fracture. This finding supports the potential significance of catastrophizing in the adjustment and adaptation to disability due to osteoporotic vertebral compression fracture regardless of pain intensity. Another limitation was that we did not assess other psychological variables that are potentially related with back pain and disability. Although recent studies identified pain catastrophizing as one of the most robust psychological predictors of the pain experience $[1,7,27]$, other psychological factors including anxiety, depression, and expectations have also been highlighted as contributors to pain experience and disability [28]. However, the present study excluded patients with a history of major depression. Finally, even though sample size $(\mathrm{n}=35)$ was calculated to achieve a power of $80 \%$ for detection of an $r^{2}=0.35$ on linear multiple regression with six predictors $(\alpha=0.05, \beta=0.2)$, this number seems to be insufficient. Therefore, our future large case series will follow this study.

In conclusion, the present study highlights that catastrophizing can contribute to disability and pain in the acute period after osteoporotic vertebral compression fracture. Therefore, in acute osteoporotic compression fracture, not only fracture treatment including brace, bed rest, and medication but also psychological support such as cognitive behavior treatment are important for relieving disability by compression fracture. As the fracture healed, however, age became the critical determinant of disability.
Therefore, in cases of osteoporotic vertebral compression fracture by a minor traumatic injury, catastrophizing would no longer influence disability after fracture healing.

\section{Conflict of interest}

The authors declare that they have no conflict of interest.

\section{Acknowledgments}

This work (Grants No. C0248313) was supported by Business for Cooperative R\&D between Industry, Academy, and Research Institute funded Korea Small and Medium Business Administration in 2014. The funder had no role in the study design, data collection and analysis, decision to publish, or preparation of the manuscript

\section{References}

[1] Sullivan MJ, Thorn B, Haythornthwaite JA, Keefe F, Martin M, Bradley LA Lefebvre JC. Theoretical perspectives on the relation between catastrophizing and pain. Clin J Pain 2001 Mar;17(1):52-64.

[2] Picavet HS, Vlaeyen JW, Schouten JS. Pain catastrophizing and kinesiophobia: predictors of chronic low back pain. Am J Epidemiol 2002 Dec 1;156(11): 1028-34.

[3] Wertli MM, Burgstaller JM, Weiser S, Steurer J, Kofmehl R, Held U. Influence of catastrophizing on treatment outcome in patients with nonspecific low back pain: a systematic review. Spine (Phila Pa 1976) 2014 Feb 1;39(3):263-73.

[4] Wertli MM, Eugster R, Held U, Steurer J, Kofmehl R, Weiser S. Catastrophizinga prognostic factor for outcome in patients with low back pain: a systematic review. Spine J 2014 Nov 1;14(11):2639-57.

[5] Vissers MM, Bussmann JB, Verhaar JA, Busschbach J, Bierma-Zeinstra SM Reijman M. Psychological factors affecting the outcome of total hip and knee arthroplasty: a systematic review. Semin Arthritis Rheum 2012 Feb;41(4) 576-88.

[6] Bergbom S, Boersma K, Overmeer T, Linton SJ. Relationship among pain catastrophizing, depressed mood, and outcomes across physical therapy treatments. Phys Ther 2011 May;91(5):754-64.

[7] Smeets RJ, Vlaeyen JW, Kester AD, Knottnerus JA. Reduction of pain catastrophizing mediates the outcome of both physical and cognitive-behavioral treatment in chronic low back pain. J Pain 2006 Apr;7(4):261-71.

[8] Dunn KM, Jordan KP, Croft PR. Contributions of prognostic factors for poor outcome in primary care low back pain patients. Eur J Pain 2011 Mar;15(3): 313-9.

[9] Khan RS, Ahmed K, Blakeway E, Skapinakis P, Nihoyannopoulos L, Macleod K, Sevdalis N, Ashrafian H, Platt M, Darzi A, Athanasiou T. Catastrophizing: a predictive factor for postoperative pain. Am J Surg 2011 Jan;201(1):122-31.

[10] Vranceanu AM, Bachoura A, Weening A, Vrahas M, Smith RM, Ring D. Psychological factors predict disability and pain intensity after skeletal trauma. J Bone Jt Surg Am 2014 Feb 5;96(3):e20.

[11] Helmerhorst GT, Vranceanu AM, Vrahas M, Smith M, Ring D. Risk factors for continued opioid use one to two months after surgery for musculoskeletal trauma. J Bone Jt Surg Am 2014 Mar 19;96(6):495-9.

[12] Denis F. The three column spine and its significance in the classification of acute thoracolumbar spinal injuries. Spine (Phila Pa 1976) 1983 Nov-Dec;8(8):817-31.

[13] Wardlaw D, Van Meirhaeghe J. Another chapter for vertebral compression fractures. Lancet 2010 Sep 25;376(9746):1031-3.

[14] Elnoamany H. Percutaneous vertebroplasty: a first line treatment in traumatic non-osteoporotic vertebral compression fractures. Asian Spine J 2015 Apr;9(2):178-84.

[15] Kim HJ, Yi JM, Cho HG, Chang BS, Lee CK, Kim JH, Yeom JS. Comparative study of the treatment outcomes of osteoporotic compression fractures without neurologic injury using a rigid brace, a soft brace, and no brace: a prospective randomized controlled non-inferiority trial. J Bone Jt Surg Am 2014 Dec 3:96(23):1959-66.

[16] Sullivan MJ, Bishop S, Pivik J. The pain catastrophizing scale: development and validation. Psychol Assess 1995 Dec;7(4):524-32.

[17] Cho S, Kim HY, Lee JH. Validation of the Korean version of the pain catastrophizing scale in patients with chronic non-cancer pain. Qual Life Res 2013 Sep;22(7):1767-72.

[18] Kim HJ, Kim SC, Kang KT, Chang BS, Lee CK, Yeom JS. Influence of educational attainment on pain intensity and disability in patients with lumbar spinal stenosis: mediation effect of pain catastrophizing. Spine (Phila Pa 1976) 2014 Feb 12;39:E637-44.

[19] Charlson ME, Pompei P, Ales KL, MacKenzie CR. A new method of classifying prognostic comorbidity in longitudinal studies: development and validation. J Chronic Dis 1987 May;40(5):373-83.

[20] Fairbank JC, Pynsent PB. The Oswestry disability Index. Spine (Philadelphia, Pa 1976) 2000 Nov 15;25(22):2940-52. 
[21] Sullivan MJ, Lynch ME, Clark AJ. Dimensions of catastrophic thinking associated with pain experience and disability in patients with neuropathic pain conditions. Pain 2005 Feb;113(3):310-5.

[22] Swinkels-Meewisse IE, Roelofs J, Oostendorp RA, Verbeek AL, Vlaeyen JW Acute low back pain: pain-related fear and pain catastrophizing influence physical performance and perceived disability. Pain 2006 Jan;120(1-2): 36-43.

[23] Kovacs FM, Seco J, Royuela A, Pena A, Muriel A, Spanish Back Pain Research N The correlation between pain, catastrophizing, and disability in subacute and chronic low back pain: a study in the routine clinical practice of the Spanish National Health Service. Spine (Phila Pa 1976) 2011 Feb 15;36(4):339-45.
[24] Carlson H, Carlson N. An overview of the management of persistent musculoskeletal pain. Ther Adv Musculoskelet Dis 2011 Apr;3(2):91-9.

[25] Civil ID, Schwab CW. The abbreviated injury scale, 1985 revision: a condensed chart for clinical use. J Trauma 1988 Jan;28(1):87-90.

[26] Das De S, Vranceanu AM, Ring DC. Contribution of kinesophobia and catastrophic thinking to upper-extremity-specific disability. J Bone Jt Surg Am 2013 Jan 2;95(1):76-81.

[27] Sullivan MJ, Neish NR. Catastrophizing, anxiety and pain during dental hygiene treatment. Community Dent Oral Epidemiol 1998 Oct;26(5):344-9.

[28] Keefe FJ, Rumble ME, Scipio CD, Giordano LA, Perri LM. Psychological aspects of persistent pain: current state of the science. J Pain 2004 May;5(4):195-211. 